ANNALES

POLONICI MATHEMATICI

$92.3(2007)$

\title{
Constructions on second order connections
}

\author{
by J. KuREK (Lublin) and W. M. Mikulski (Kraków)
}

\begin{abstract}
We classify all $\mathcal{F} \mathcal{M}_{m, n}$-natural operators $\mathcal{D}: J^{2} \rightsquigarrow J^{2} V^{A}$ transforming second order connections $\Gamma: Y \rightarrow J^{2} Y$ on a fibred manifold $Y \rightarrow M$ into second order connections $\mathcal{D}(\Gamma): V^{A} Y \rightarrow J^{2} V^{A} Y$ on the vertical Weil bundle $V^{A} Y \rightarrow M$ corresponding to a Weil algebra $A$.
\end{abstract}

0. Introduction. An $r$ th order connection on a fibred manifold $Y \rightarrow M$ is a section $\Gamma: Y \rightarrow J^{r} Y$ of the $r$-jet prolongation $J^{r} Y \rightarrow Y$ of $Y \rightarrow M$ (see [5]). In [6], we studied the problem how a first order connection $\Gamma$ : $Y \rightarrow J^{1} Y$ on $Y \rightarrow M$ induces a first order connection $\mathcal{D}(\Gamma): V^{A} Y \rightarrow$ $J^{1} V^{A} Y$ on the vertical Weil bundle $V^{A} Y \rightarrow M$ corresponding to a Weil algebra $A$. In the present paper we study the similar problem of how a second order connection $\Gamma: Y \rightarrow J^{2} Y$ on a fibred manifold $Y \rightarrow M$ can induce a second order connection $\mathcal{D}(\Gamma): V^{A} Y \rightarrow J^{2} V^{A} Y$ on $V^{A} Y \rightarrow M$. This problem corresponds to the classification of $\mathcal{F} \mathcal{M}_{m, n}$-natural operators $\mathcal{D}: J^{2} \rightsquigarrow J^{2} V^{A}$ in the sense of [5], where $\mathcal{F} \mathcal{M}_{m, n}$ is the category of fibred manifolds with $n$-dimensional fibres and $m$-dimensional bases and their fibred local diffeomorphisms. We prove that the set of all $\mathcal{F} \mathcal{M}_{m, n}$-natural operators $\mathcal{D}: J^{2} \rightsquigarrow J^{2} V^{A}$ forms a $\operatorname{dim}_{\mathbb{R}} A$-dimensional affine space and we explicitly describe this affine space. Thus we obtain a quite different result than the one from [6], where it is proved that there is only one $\mathcal{F M}_{m, n^{-}}$ natural operator $\mathcal{D}: J^{1} \rightsquigarrow J^{1} V^{A}$.

All manifolds and maps are of class $C^{\infty}$.

1. The main result. The general concept of natural operators is described in [5]. In particular, an $\mathcal{F} \mathcal{M}_{m, n}$-natural operator $\mathcal{D}: J^{r} \rightsquigarrow J^{r} V^{A}$ transforming $r$ th order general connections $\Gamma$ on $\mathcal{F} \mathcal{M}_{m, n}$-objects $Y \rightarrow M$ to $r$ th order connections $\mathcal{D}(\Gamma)$ on the vertical Weil bundle $V^{A} Y \rightarrow M$

2000 Mathematics Subject Classification: 58A05, 58A20.

Key words and phrases: higher order connection, (vertical) Weil bundle, natural operator. 
corresponding to a Weil algebra $A$ is a family of $\mathcal{F} \mathcal{M}_{m, n}$-invariant regular operators (functions) $\mathcal{D}: \operatorname{Con}^{r}(Y \rightarrow M) \rightarrow \operatorname{Con}^{r}\left(V^{A} Y \rightarrow M\right)$ from the space $\operatorname{Con}^{r}(Y \rightarrow M)$ of all $r$ th order connections on $Y \rightarrow M$ into the space $\operatorname{Con}^{r}\left(V^{A} Y \rightarrow M\right)$ of all $r$ th order connections on $V^{A} Y \rightarrow M$ for $\mathcal{F M}_{m, n}$-objects $Y \rightarrow M$. By [6], any $\mathcal{F M}_{m, n}$-natural operator $\mathcal{D}$ : $J^{1} \rightsquigarrow J^{1} V^{A}$ is equal to the well-known $A$-vertical prolongation operator $\mathcal{V}^{A}: J^{1} \rightsquigarrow J^{1} V^{A}$. We have the following examples of $\mathcal{F} \mathcal{M}_{m, n}$-natural operators $\mathcal{D}: J^{2} \rightsquigarrow J^{2} V^{A}$.

EXAMPLE 1. Given a general second order connection $\Gamma: Y \rightarrow J^{2} Y$ on $Y \rightarrow M$ we define a second order general connection $\mathcal{V}^{A, 2} \Gamma$ on $V^{A} Y \rightarrow M$ by $\mathcal{V}^{A, 2} \Gamma=\left(\kappa^{A, 2}\right)_{Y} \circ V^{A} \Gamma: V^{A} Y \rightarrow J^{2} V^{A} Y$, where $\left(\kappa^{A, 2}\right)_{Y}: V^{A} J^{2} Y \rightarrow$ $J^{2} V^{A} Y$ is the canonical exchange isomorphism [5], [1]. The correspondence $\mathcal{V}^{A, 2}: J^{2} \rightsquigarrow J^{2} V^{A}$ is the $\mathcal{F} \mathcal{M}_{m, n}$-natural operator in question.

To give the next such example we need some preparation. Let $\Gamma: Y \rightarrow$ $J^{2} Y$ be a second order connection on $Y \rightarrow M$ with first order underlying connection $\Gamma^{0}: Y \rightarrow J^{1} Y$. Let $\Gamma^{0} * \Gamma^{0}:=J^{1} \Gamma^{0} \circ \Gamma^{0}: Y \rightarrow \bar{J}^{2} Y$ be the second order semi-holonomic Ehresmann prolongation of $\Gamma^{0}$ and $C^{(2)}: \bar{J}^{2} Y \rightarrow J^{2} Y$ be the well-known symmetrization of second order semiholonomic jets [4], [3]. Then $\left(\Gamma^{0}\right)^{2}:=C^{(2)} \circ\left(\Gamma^{0} * \Gamma^{0}\right): Y \rightarrow J^{2} Y$ is another second order connection on $Y \rightarrow M$ with the same underlying first order connection $\Gamma^{0}$. Since $J^{2} Y \rightarrow J^{1} Y$ is an affine bundle with corresponding vector bundle $S^{2} T^{*} M \otimes V Y$ over $J^{1} Y$, we have the difference tensor field $\mathcal{E}(\Gamma):=\Gamma-\left(\Gamma^{0}\right)^{2}: Y \rightarrow S^{2} T^{*} M \otimes V Y$. Using this tensor, we construct the next example.

EXAMPLE 2. For any $a \in A$ we have a tensor field $\mathcal{E}^{a}(\Gamma): V^{A} Y \rightarrow$ $S^{2} T^{*} M \otimes V V^{A} Y$ given by $\left.\mathcal{E}^{a}(\Gamma)\left(X_{1}, X_{2}\right)\right):=J_{a} \circ \mathcal{V}^{A}\left(\mathcal{E}(\Gamma)\left(X_{1}, X_{2}\right)\right)$, where $J_{a}: V V^{A} Y \rightarrow V V^{A} Y$ is a canonical "affinor" defined fibre-wise from the canonical affinor $J_{a}: T T^{A} N \rightarrow T T^{A} N$, and $\mathcal{V}^{A}\left(\mathcal{E}(\Gamma)\left(X_{1}, X_{2}\right)\right)$ is the flow prolongation of the vertical vector field $\mathcal{E}(\Gamma)\left(X_{1}, X_{2}\right)$ to $V^{A} Y$ for any vector fields $X_{1}, X_{2}$ on $M$. Since $J^{2} V^{A} Y \rightarrow J^{1} V^{A} Y$ is an affine bundle with the corresponding vector bundle $S^{2} T^{*} M \otimes V V^{A} Y$ over $J^{1} V^{A} Y$, we can define a second order connection $\mathcal{D}^{a}(\Gamma): \mathcal{V}^{A, 2} \Gamma+\mathcal{E}^{a}(\Gamma)$ on $V^{A} Y \rightarrow M$. The correspondence $\mathcal{D}^{a}: J^{2} \rightsquigarrow J^{2} V^{A}$ is an $\mathcal{F} \mathcal{M}_{m, n}$-natural operator.

The main result of the paper is the following classification theorem.

TheOREM 1. Every $\mathcal{F} \mathcal{M}_{m, n}$-natural operators $\mathcal{D}: J^{2} \rightsquigarrow J^{2} V^{A}$ is $\mathcal{D}^{a}$ : $J^{2} \rightsquigarrow J^{2} V^{A}$ for some $a \in A$.

The proof of Theorem 1 will occupy the rest of the paper. We prove three propositions. In Proposition 1, we show that any $\mathcal{F} \mathcal{M}_{m, n}$-natural operator $\mathcal{D}: J^{2} \rightsquigarrow J^{2} V^{A}$ is of finite order. In Proposition 2, we observe that for any $\mathcal{F} \mathcal{M}_{m, n}$-natural operator $\mathcal{D}: J^{2} \rightsquigarrow J^{2} V^{A}$ the under- 
lying first order connection $\mathcal{D}(\Gamma)^{0}$ of $\mathcal{D}(\Gamma)$ on $V^{A} Y \rightarrow M$ is equal to the connection $\mathcal{V}^{A} \Gamma^{0}$, where $\Gamma^{0}$ is the underlying first order connection of the second order connection $\Gamma: Y \rightarrow J^{2} Y$ on $Y \rightarrow M$. Thus we have the difference $\mathcal{F} \mathcal{M}_{m, n}$-natural operator $\mathcal{E}: J^{2} \rightsquigarrow S^{2} T^{*} \otimes V V^{A}$ given by $\mathcal{E}(\Gamma)=\mathcal{D}(\Gamma)-\mathcal{V}^{A, 2} \Gamma: V^{A} Y \rightarrow S^{2} T^{*} M \otimes V V^{A} Y$. In Proposition 3, we prove that the vector space (over $\mathbb{R}$ ) of all $\mathcal{F} \mathcal{M}_{m, n}$-natural operators $\mathcal{E}: J^{2} \rightsquigarrow S^{2} T^{*} \otimes V V^{A}$ is of dimension $\leq \operatorname{dim}_{\mathbb{R}} A$. Then Theorem 1 follows by a dimension argument.

1. Finite order. We start the proof of Theorem 1 from the following proposition.

Proposition 1. Any $\mathcal{F} \mathcal{M}_{m, n}$-natural operator $\mathcal{D}$ transforming second order general connections $\Gamma$ on $Y \rightarrow M$ into second order general connections $\mathcal{D}(\Gamma)$ on $V^{A} Y \rightarrow M$ is of finite order.

Proof. (See also the proof of Proposition 3 in [6].) This follows from the proof of Proposition 23.7 in [5], which can be generalized to our situation in the following way. Let $x^{i}, y^{j}(i=1, \ldots, m, j=1, \ldots, n)$ be the usual fibre coordinates on $\mathbb{R}^{m, n}$, the trivial bundle $\mathbb{R}^{m} \times \mathbb{R}^{n} \rightarrow \mathbb{R}^{m}$. Let $x^{i}, y_{\alpha}^{j}$ for $\alpha \in(\mathbb{N} \cup\{0\})^{m}$ with $0 \leq|\alpha| \leq 2$ be the induced coordinates on $J^{2} \mathbb{R}^{m, n}$. Consider the map $\varphi_{a, b}: \mathbb{R}^{m, n} \rightarrow \mathbb{R}^{m, n}, \varphi_{a, b}(x, y)=(a x, b y)$. Fix some $r \in \mathbb{N}$ and choose $a=b^{-r}, 0<b<1$ arbitrary. Hence for every multiindex $\alpha=\alpha_{1}+\alpha_{2}$, where $\alpha_{1}$ includes all the derivatives with respect to the base coordinates while $\alpha_{2}$ those with respect to the fibre coordinates, and for every second order general connection $\Gamma$ on $\mathbb{R}^{m, n}$,

$$
\left|\partial^{\alpha_{1}+\alpha_{2}}\left(y_{\beta}^{j} \circ \varphi_{a, b}^{*} \Gamma\right)(0,0)\right|=b^{r\left(|\beta|+\left|\alpha_{1}\right|\right)+1-\left|\alpha_{2}\right|}\left|\partial^{\alpha_{1}+\alpha_{2}}\left(y_{\beta}^{j} \circ \Gamma\right)(0,0)\right|
$$

for all $|\beta|=1,2$, and so for all $|\alpha| \leq r$ we get

$$
\left|\partial^{\alpha}\left(\varphi_{a, b}^{*} \Gamma\right)(0,0)\right| \leq b\left|\partial^{\alpha} \Gamma(0,0)\right|,
$$

where $\left|\partial^{\alpha} \Gamma(0,0)\right|=\sum_{j=1}^{n} \sum_{|\beta|=1,2}\left|\partial^{\alpha}\left(y_{\beta}^{j} \circ \Gamma\right)(0,0)\right|$. On the other hand, there is a compact subset $K \subset\left(V^{A} \mathbb{R}^{m, n}\right)_{(0,0)}=T_{0}^{A} \mathbb{R}^{n}$ ( $K$ is a compact neighbourhood of $\left.z_{0}=j^{A} 0\right)$ such that for any $z \in\left(V^{A} \mathbb{R}^{m, n}\right)_{(0,0)}$ we will have $V^{A} \varphi_{a, b}(z) \in K$ for sufficiently small $b$. Hence Corollary 23.4 in [5] implies our assertion.

2. An underlying connection. Given a second order general connection $\Gamma: Y \rightarrow J^{2} Y$ on $Y \rightarrow M$ we denote by $\Gamma^{0}: Y \rightarrow J^{1} Y$ the underlying first order general connection on $Y \rightarrow M$.

Proposition 2. Let $\mathcal{D}$ be an $\mathcal{F} \mathcal{M}_{m, n}$-natural operator transforming second order general connections $\Gamma$ on $Y \rightarrow M$ into second order general con- 
nections $\mathcal{D}(\Gamma)$ on $V^{A} Y \rightarrow M$. Then

$$
(\mathcal{D}(\Gamma))^{0}=\left(\mathcal{V}^{A, 2} \Gamma\right)^{0}
$$

for any second order general connection $\Gamma$ on $Y \rightarrow M$.

Proof. Let $x^{i}, y^{j}, y_{\alpha}^{j}$ be as in the proof of Proposition 1 . Let $v^{l}$ be a coordinate system on $A^{n}$. Then on $J_{0}^{1}\left(\mathbb{R}^{m}, A^{n}\right)$ we have the induced coordinates $v^{l}, v_{k}^{l}$, where $l=1, \ldots, \operatorname{dim} A^{n}, k=1, \ldots, m$. Let $\Gamma$ be a second order general connection on $\mathbb{R}^{m, n}$. We will study $(\mathcal{D}(\Gamma))_{w}^{0} \in\left(J^{1} V^{A} \mathbb{R}^{m, n}\right)_{0}=$ $J_{0}^{1}\left(\mathbb{R}^{m}, A^{n}\right)$ for $w \in\left(V^{A} \mathbb{R}^{m, n}\right)_{(0,0)}=\left(T^{A} \mathbb{R}^{n}\right)_{0}$.

We fix an arbitrary $w$ as above. By Proposition $1, \mathcal{E}$ is of finite order $q$. So, we can assume that $y_{\alpha}^{j} \circ \Gamma$ is a polynomial of degree $q$ for any $j, \alpha$ as above, i.e. $y_{\alpha}^{j} \circ \Gamma(x, y)=\sum \Gamma_{\alpha, \beta, \varrho}^{j} x^{\beta} y^{\varrho}$ for $(x, y) \in \mathbb{R}^{m, n}$, where the sum is over all $\beta \in(\mathbb{N} \cup\{0\})^{m}$ and $\varrho \in(\mathbb{N} \cup\{0\})^{n}$ with $|\beta|+|\varrho| \leq q$, and $\Gamma_{\alpha, \beta, \varrho}^{j}$ are real numbers determined by $\Gamma$. Moreover, we have $y_{(0)}^{j} \circ \Gamma(x, y)=y^{j}$. We identify $\Gamma$ with $\left(\Gamma_{\alpha, \beta, \varrho}^{j}\right)$. Using the invariance of $\mathcal{D}$ with respect to the base homotheties $t \operatorname{id}_{\mathbb{R}^{m}} \times \mathrm{id}_{\mathbb{R}^{n}}$ we obtain the homogeneity conditions

$$
v^{l} \circ\left(\mathcal{D}\left(t^{|\alpha|+|\beta|} \Gamma_{\alpha, \beta, \varrho}^{j}\right)\right)_{w}^{0}=v^{l} \circ\left(\mathcal{D}\left(\Gamma_{\alpha, \beta, \varrho}^{j}\right)\right)_{w}^{0}
$$

and

$$
v_{k}^{l} \circ\left(\mathcal{D}\left(t^{|\alpha|+|\beta|} \Gamma_{\alpha, \beta, \varrho}^{j}\right)\right)_{w}^{0}=t v_{k}^{l} \circ\left(\mathcal{D}\left(\Gamma_{\alpha, \beta, \varrho}^{j}\right)\right)_{w}^{0} .
$$

Then by the homogeneous function theorem, $(\mathcal{D}(\Gamma))_{w}^{0}$ is independent of $\Gamma_{\alpha, \beta, \varrho}^{j}$ for $|\alpha|=2$. This means that $(\mathcal{D}(\Gamma))^{0}$ over $(0,0) \in \mathbb{R}^{m} \times \mathbb{R}^{n}$ depends on a finite jet of $\Gamma^{0}$ at $(0,0)$ only. Then we have a well-defined $\mathcal{F} \mathcal{M}_{m, n}$-natural operator $\mathcal{D}^{0}$ by $\mathcal{D}^{0}(\widetilde{\Gamma})=(\mathcal{D}(\Gamma))^{0}$ for any first order general connection $\widetilde{\Gamma}$ on $Y \rightarrow M$, where $\Gamma$ is a second order general connection on $Y \rightarrow M$ with $\Gamma^{0}=\widetilde{\Gamma}$. By the above-mentioned result of $[6], \mathcal{D}^{0}=\mathcal{V}^{A}$. This implies the equality in the proposition.

3. The main difficulty. The main difficulty in the proof of Theorem 1 is to establish the following proposition.

Proposition 3. The vector space over $\mathbb{R}$ of all $\mathcal{F} \mathcal{M}_{m, n}$-natural operators sending second order general connections $\Gamma$ on $Y \rightarrow M$ into tensor fields $\mathcal{E}(\Gamma): V^{A} Y \rightarrow S^{2} T^{*} M \otimes V V^{A} Y$ is of dimension $\leq \operatorname{dim}_{\mathbb{R}} A$.

To prove Proposition 3 we need some lemmas.

Let $x^{i}, y^{j}, y_{\alpha}^{j}$ and $v^{l}$ be as in the proof of Proposition 2. We can of course assume that the $v^{l}$ are obtained as follows. We choose a basis $a_{1}, \ldots, a_{K}$ of $A$ over $\mathbb{R}$. Let $\left(a_{1}, 0, \ldots, 0\right), \ldots,\left(a_{K}, 0, \ldots, 0\right),\left(0, a_{K+1}, 0, \ldots, 0\right), \ldots$, $\left(0, \ldots, 0, a_{n K}\right)$ be the corresponding basis of $A^{n}$. Then $v^{l}, l=1, \ldots, \operatorname{dim} A^{n}$, is the basis dual to the last one. Let $\mathcal{E}$ be an $\mathcal{F} \mathcal{M}_{m, n}$-natural operator transforming second order connections $\Gamma$ on $Y \rightarrow M$ into tensor fields 
$\mathcal{E}(\Gamma): V^{A} Y \rightarrow S^{2} T^{*} M \otimes V V^{A} Y$. We denote the order of $\mathcal{E}$ by $q$ ( $q$ is finite by Proposition 1).

LEMMA 1. If

$$
\left\langle\mathcal{E}(\Gamma)_{w}, u \odot u\right\rangle=0 \in V_{w} V^{A} \mathbb{R}^{m, n}=T_{w} T^{A} \mathbb{R}^{n}=T_{w} A^{n}
$$

for all $w \in\left(V^{A} \mathbb{R}^{m, n}\right)_{(0,0)}=T_{0}^{A} \mathbb{R}^{n}$, all $u \in T_{0} \mathbb{R}^{m}$ and all second order general connections $\Gamma$ on $\mathbb{R}^{m, n}$, then $\mathcal{E}=0$.

Proof. This is an immediate consequence of the invariance of $\mathcal{E}$ with respect to charts.

Using the invariance of $\mathcal{E}$ with respect to $\mathcal{F} \mathcal{M}_{m, n}$-maps of the form $\varphi \times \operatorname{id}_{\mathbb{R}^{n}}$ for linear isomorphisms $\varphi: \mathbb{R}^{m} \rightarrow \mathbb{R}^{m}$, we have

LEMMA 2. If

$$
\left\langle\mathcal{E}(\Gamma)_{w}, u_{0} \odot u_{0}\right\rangle=0 \in T_{w} A^{n}
$$

for all $w \in T_{0}^{A} \mathbb{R}^{n}$ and all second order general connections $\Gamma$ on $\mathbb{R}^{m, n}$, where $u_{0}:=\frac{\partial}{\partial x^{1}}{ }_{\mid 0}$, then $\mathcal{E}=0$.

Define

$$
\Phi_{w}^{l}(\Gamma):=d_{w} v^{l}\left(\left\langle\mathcal{E}(\Gamma)_{w}, u_{0} \odot u_{0}\right\rangle\right) \in \mathbb{R}
$$

for all $w \in T_{0}^{A} \mathbb{R}^{n}$, all second order connections $\Gamma$ on $\mathbb{R}^{m, n}$ and $l=1, \ldots$, $\operatorname{dim} A^{n}$.

LEMMA 3. If $\Phi_{w}^{l}(\Gamma)=0$ for all $w$ and $\Gamma$ as above and $l=1, \ldots, \operatorname{dim} A$, then $\mathcal{E}=0$.

Proof. Because of the invariance of $\mathcal{E}$ with respect to permutations of the fibred coordinates, from the assumption of the lemma we deduce that $\Phi_{w}^{l}(\Gamma)=0$ for all $w$ and $\Gamma$ as above and $l=\operatorname{dim} A^{n}$. Then $\left\langle\mathcal{E}(\Gamma)_{w}, u_{0} \odot u_{0}\right\rangle$ $=0$ for all $w$ and $\Gamma$ as, and Lemma 2 ends the proof.

Because of the order of $\mathcal{E}$ we can assume that in the above lemmas we have

$$
y_{\alpha}^{j} \circ \Gamma(x, y)=\sum \Gamma_{\alpha, \beta, \varrho}^{j} x^{\beta} y^{\varrho}
$$

for all $(x, y) \in \mathbb{R}^{m, n}$, where the sum is over all $\beta \in(\mathbb{N} \cup\{0\})^{m}$ and $\varrho \in$ $(\mathbb{N} \cup\{0\})^{n}$ with $|\beta|+|\varrho| \leq q$, and $\Gamma_{\alpha, \beta, \varrho}^{j}$ are real numbers determined by $\Gamma$. Moreover, we have $y_{(0)}^{j} \circ \Gamma(x, y)=y^{j}$.

We identify $\Gamma$ with $\left(\Gamma_{\alpha, \beta, \varrho}^{j}\right)$. Using the invariance of $\mathcal{E}$ with respect to the base homotheties $\left(t^{1} x^{1}, \ldots, t^{m} x^{m}, y^{1}, \ldots, y^{n}\right)$ for $t^{j}>0$, we get the homogeneity condition

$$
\left(t^{1}\right)^{2} \Phi_{w}^{l}\left(\Gamma_{\alpha, \beta, \varrho}^{j}\right)=\Phi_{w}^{l}\left(t^{\alpha+\beta} \Gamma_{\alpha, \beta, \varrho}^{j}\right)
$$


Then by the homogeneous function theorem we can write

$$
\begin{aligned}
\Phi_{w}^{l}(\Gamma)= & \sum a_{j}^{\varrho} \Gamma_{(2,0, \ldots, 0),(0), \varrho}^{j}+\sum b_{j}^{\varrho} \Gamma_{(1,0 \ldots, 0),(1,0, \ldots, 0), \varrho}^{j} \\
& +\sum c_{j_{1}, j_{2}}^{\varrho_{1}, \varrho_{2}} \Gamma_{(1,0, \ldots, 0),(0), \varrho_{1}}^{j_{1}} \Gamma_{(1,0, \ldots, 0),(0), \varrho_{2}}^{j_{2}}
\end{aligned}
$$

for some uniquely determined real numbers $a_{j}^{\varrho}=a_{j}^{\varrho, l}(w), b_{j}^{\varrho}=b_{j}^{\varrho, l}(w)$ and $c_{j_{1}, j_{2}}^{\varrho_{1}, \varrho_{2}}=c_{j_{1}, j_{2}}^{\varrho_{1}, l}(w)$ (smoothly depending on $w$ ), where the first sum is over all $j=1, \ldots, n$ and all $\varrho \in(\mathbb{N} \cup\{0\})^{n}$ with $|\varrho| \leq q$, the second sum is over all $j=1, \ldots, n$ and all $\varrho \in(\mathbb{N} \cup\{0\})^{n}$ with $|\varrho| \leq q-1$ and the third sum is over all $\left(\varrho_{1}, j_{1}\right) \leq\left(\varrho_{2}, j_{2}\right)$ for $j_{1}, j_{2}=1, \ldots, n$ and $\varrho_{1}, \varrho_{2} \in$ $(\mathbb{N} \cup\{0\})^{n}$ with $\left|\varrho_{1}\right| \leq q$ and $\left|\varrho_{2}\right| \leq q$ (here $\leq$ means an ordering). Of course $(2,0, \ldots, 0),(1,0, \ldots, 0),(0) \in(\mathbb{N} \cup\{0\})^{m}$.

Lemma 4. Assume that all $a_{j}^{\varrho}$, all $b_{j}^{\varrho}$ and all $c_{j_{1}, j_{2}}^{\varrho_{1}, \varrho_{2}}$ defined by $(*)$ are 0 for all $w \in T_{0}^{A} \mathbb{R}^{n}$ and all $l=1, \ldots, \operatorname{dim} A$. Then $\mathcal{E}=0$.

Proof. This is obvious in view of the previous lemma.

Lemma 5. We have

$$
a_{j}^{\varrho}+b_{j}^{\varrho}=0
$$

for all $j, \varrho, w$ in question and $l=1, \ldots, \operatorname{dim} A$.

Proof. Fix $\varrho_{0}$ and $j_{0}$. Choose $\Gamma=\left(\Gamma_{\alpha, \beta, \varrho}^{j}\right)$ such that $\Gamma_{(1,0, \ldots, 0),(0), \varrho_{0}}^{j_{0}}=1$, and $\Gamma_{\alpha, \beta, \varrho}^{j}=0$ for other $(j, \alpha, \beta, \varrho)$ with $|\alpha| \geq 1$. Let $\varphi=\left(x^{1}+\frac{1}{2}\left(x^{1}\right)^{2}\right.$, $\left.x^{2}, \ldots, x^{m}, y^{1}, \ldots, y^{n}\right)^{-1}$. Using the invariance of $\mathcal{E}$ with respect to $\varphi$ we have

$$
\Phi_{w}^{l}\left(\varphi_{*} \Gamma\right)=\Phi_{w}^{l}(\Gamma)
$$

because $\varphi$ preserves $w, v^{l}$ and $u_{0} \odot u_{0}$. Set

$$
\Phi_{w}^{l}(\Gamma)=a .
$$

We have $j_{(0,0)}^{q}\left(\varphi_{*} \Gamma\right)=\left(\widetilde{\Gamma}_{\alpha, \beta, \varrho}^{j}\right)$, where

$$
\widetilde{\Gamma}_{(1,0, \ldots, 0),(0), \varrho_{0}}^{j_{0}}=1, \quad \widetilde{\Gamma}_{(2,0, \ldots, 0),(0), \varrho_{0}}^{j_{0}}=1, \quad \widetilde{\Gamma}_{(1,0, \ldots, 0),(1,0, \ldots, 0), \varrho_{0}}^{j_{0}}=1
$$

and other $\widetilde{\Gamma}_{\alpha, \beta, \varrho}^{j}$ are zero for $|\alpha|=1,2$. (Indeed,

$$
\Gamma(z, y)=j_{z}^{2}\left(y+y^{\varrho_{0}}\left(x^{1}-z^{1}\right) e_{j_{0}}\right) \in J_{z}^{2}\left(\mathbb{R}^{m, n}\right)_{y},
$$

where $\left\{e_{j}\right\}$ is the canonical basis in $\mathbb{R}^{n}$. Then

$$
\left(\varphi_{*} \Gamma\right)(z, y)=j_{z}^{2}\left(y+y^{\varrho_{0}}\left(x^{1}+\frac{1}{2}\left(x^{1}\right)^{2}-z^{1}-\frac{1}{2}\left(z^{1}\right)^{2}\right) e_{j_{0}}\right) .
$$

This implies the formulas.) Then

$$
\Phi_{w}^{l}\left(\varphi_{*} \Gamma\right)=a_{j_{0}}^{\varrho_{0}}+b_{j_{0}}^{\varrho_{0}}+a .
$$

Hence $a_{j_{0}}^{\varrho_{0}}+b_{j_{0}}^{\varrho_{0}}=0$. 
Lemma 6. Suppose that all $a_{j}^{\varrho}$ defined by $(*)$ are zero for any $w$ in question and $l=1, \ldots, \operatorname{dim} A$. Then $\mathcal{E}=0$.

Proof. By assumption and Lemma 5 , all $b_{j}^{\varrho}$ are zero. Then it is sufficient to show that $c_{j_{1}, j_{2}}^{\varrho_{1}, \varrho_{2}}=0$ for all $\varrho_{1}, \varrho_{2}, j_{1}, j_{2}, w$ and $l$ in question.

Fix $\varrho_{1}, \varrho_{2}, j_{1}, j_{2}, l, w$. Let $a, b \in \mathbb{R}$. Let $\Gamma^{0}$ be the trivial second order connection on $\mathbb{R}^{m, n}$ given by

$$
\Gamma^{0}(z, y)=j_{z}^{2}(y) \in J_{z}^{2}\left(\mathbb{R}^{m, n}\right)_{y}
$$

Then by $(*)$ we have

$$
\Phi_{w}^{l}\left(\Gamma^{0}\right)=0
$$

Choose an $\mathcal{F} \mathcal{M}_{m, n}$-map $\psi: \mathbb{R}^{m, n} \rightarrow \mathbb{R}^{m, n}$ given by

$$
\psi(x, y)=\left(x, y+a x^{1} y^{\varrho_{1}} e_{j_{1}}+b x^{1} y^{\varrho_{2}} e_{j_{2}}\right) .
$$

Then

$$
\Phi_{w}^{l}\left(\psi_{*} \Gamma^{0}\right)=0
$$

because of the invariance of $\mathcal{E}, u_{0}, w$ and $v^{l}$ with respect to $\psi$. Write $\psi^{-1}(x, y)=(x, \widetilde{y})$. Then

$$
\left(\psi_{*} \Gamma^{0}\right)(z, y)=j_{z}^{2}\left(\widetilde{y}+a x^{1} \widetilde{y}^{\varrho_{1}} e_{j_{1}}+b x^{1} \widetilde{y}^{\varrho_{2}} e_{j_{2}}\right) .
$$

Then by $(*), \Phi_{w}^{l}\left(\psi_{*} \Gamma^{0}\right)$ is a polynomial in $a$ and $b$ with the coefficient of $a b$ equal to $c_{j_{1}, j_{2}}^{\varrho_{1}, \varrho_{2}}$ as all $b_{j}^{\varrho}$ are zero. Therefore $c_{j_{1}, j_{2}}^{\varrho_{1}, \varrho_{2}}=0$.

Lemma 7. Suppose that all $a_{j}^{(0)}$ defined by $(*)$ are zero for any $w$ in question and $l=1, \ldots, \operatorname{dim} A$. Then $\mathcal{E}=0$.

Proof. For any $\varrho \in(\mathbb{N} \cup\{0\})^{n}$ and $j=1, \ldots, n$, let $\Gamma^{\varrho, j}$ be the second order connection on $\mathbb{R}^{m, n}$ given by

$$
\Gamma^{\varrho, j}(z, y)=j_{z}^{2}\left(y+\left(x^{1}-z^{1}\right)^{2} y^{\varrho} e_{j}\right), \quad(z, y) \in \mathbb{R}^{m, n} .
$$

By $(*)$ and the assumption of the lemma we have

$$
d_{w} v^{l} \circ\left\langle\mathcal{E}\left(\Gamma^{(0), j}\right)(w), u_{0} \odot u_{0}\right\rangle=\Phi_{w}^{l}\left(\Gamma^{(0), j}\right)=a_{j}^{(0), l}(w)=0
$$

for any $w \in\left(V^{A} \mathbb{R}^{m, n}\right)_{(0,0)}, j=1, \ldots, n$ and $l=1, \ldots, \operatorname{dim}_{\mathbb{R}} A$. Then by the invariance of $\mathcal{E}$ with respect to the permutations of fibred coordinates we have

$$
d_{w} v^{l} \circ\left\langle\mathcal{E}\left(\Gamma^{(0), j}\right)(w), u_{0} \odot u_{0}\right\rangle=0
$$

for any $w \in\left(V^{A} \mathbb{R}^{m, n}\right)_{(0,0)}, j=1, \ldots, n$ and $l=1, \ldots, \operatorname{dim}_{\mathbb{R}} A^{n}$. Therefore

$$
\left\langle\mathcal{E}\left(\Gamma^{(0), j}\right)(w), u_{0} \odot u_{0}\right\rangle=0
$$

for any $w \in\left(V^{A} \mathbb{R}^{m, n}\right)_{(0,0)}$ and $j=1, \ldots, n$. Let $\varrho \in(\mathbb{N} \cup\{0\})^{n}, 1 \leq|\varrho| \leq q$, $j=1, \ldots, n$. Let $\tau \in \mathbb{R}$ be sufficiently small. Consider an $\mathcal{F} \mathcal{M}_{m, n}$-map 
$\varphi^{\varrho j \tau}: \mathbb{R}^{m, n} \rightarrow \mathbb{R}^{m, n}, \varphi^{\varrho j \tau}(x, y)=\left(x, y+\tau y^{\varrho+1_{j}} e_{j}\right)$ (defined near $\left.(0,0)\right)$. We see that

$$
\left(\varphi_{*}^{\varrho j \tau} \Gamma^{(0), j}\right)(z, y)=j_{z}^{2}\left(y+\left(x^{1}-z^{1}\right)^{2} e_{j}+\tau\left(\varrho_{j}+1\right)\left(x^{1}-z^{1}\right)^{2} y^{\varrho} e_{j}+\cdots\right) .
$$

Then using the invariance of $\mathcal{E}$ with respect to $\varphi^{\varrho j \tau}$ we get

$$
\left\langle\mathcal{E}\left(\varphi_{*}^{\varrho j \tau} \Gamma^{(0), j}\right)(w), u_{0} \odot u_{0}\right\rangle=0
$$

for all $w \in\left(V^{A} \mathbb{R}^{m, n}\right)_{(0,0)}$. The left hand side of the last formula is a polynomial in $\tau$. The coefficient of $\tau=\tau^{1}$ in this polynomial is $\left(\varrho_{j}+1\right)\left\langle\mathcal{E}\left(\Gamma^{\varrho, j}\right)(w), u_{0} \odot u_{0}\right\rangle$. Then $\left\langle\mathcal{E}\left(\Gamma^{\varrho, j}\right)(w), u_{0} \odot u_{0}\right\rangle=0$ for any $w \in$ $\left(V^{A} \mathbb{R}^{m, n}\right)_{(0,0)}$. Thus $a_{j}^{\varrho}=0$ for all $l$ and $w$ in question. Then $\mathcal{E}=0$ by Lemma 6.

Lemma 8. Suppose that all $a_{j}^{(0)}$ defined by $(*)$ are zero for $l=$ $1, \ldots, \operatorname{dim} A$ and $w=0 \in T_{0}^{A} \mathbb{R}^{n}$. Then $\mathcal{E}=0$.

Proof. By $(*)$ we have

$$
\Phi_{w}^{l}\left(\Gamma^{(0), j}\right)=a_{j}^{(0)}=a_{j}^{(0), l}(w),
$$

where $\Gamma^{(0), j}$ is defined in the proof of Lemma 7 . Let $h_{t}=\operatorname{id}_{\mathbb{R}^{m}} \times t \operatorname{id}_{\mathbb{R}^{n}}$ be the fibre homothety. Then $\left(\left(h_{t}\right)_{*} \Gamma^{(0), j}\right)(z, y)=j_{z}^{2}\left(y+t\left(x^{1}-z^{1}\right)^{2} e_{j}\right)$, and then by $(*)$ we have

$$
\Phi_{t w}^{l}\left(\left(h_{t}\right)_{*} \Gamma^{(0), j}\right)=t a_{j}^{(0), l}(t w) .
$$

Hence by the invariance of $\mathcal{E}$ with respect to the fibre homotheties $h_{t}$ we get $\Phi_{t w}^{l}\left(\left(h_{t}\right)_{*} \Gamma^{(0), j}\right)=t \Phi_{w}^{l}\left(\Gamma^{(0), j}\right)$, and therefore

$$
a_{j}^{(0), l}(w)=a_{j}^{(0), l}(t w) .
$$

Then putting $t \rightarrow 0$ and using the assumption of the lemma we see that $a_{j}^{(0)}=0$ for any $l$ and $w$ in question. Then Lemma 7 ends the proof.

Lemma 9. Suppose that $a_{1}^{(0)}=0$ for $w=0 \in T_{0}^{A} \mathbb{R}^{n}$ and $l=$ $1, \ldots, \operatorname{dim} A$. Then $\mathcal{E}=0$.

Proof. Let $a_{t}=\left(x^{1}, t x^{2}, \ldots, t x^{m}, y^{1}, \ldots, y^{n}\right)$ for $t \neq 0$ be $\mathcal{F} \mathcal{M}_{m, n}$-maps. Clearly, $\left(\left(a_{t}\right)_{*} \Gamma^{(0), j}\right)(y, z)=j_{z}^{2}\left(y+t\left(x^{1}-z^{1}\right)^{2} e_{j}\right)$ for $j=2, \ldots, n$. Then by $(*)$ and the invariance of $\mathcal{E}$ with respect to $a_{t}$ we get

$$
a_{j}^{(0), l}(0)=t a_{j}^{(0), l}(0)
$$

for $j=2, \ldots, n$. Then $a_{j}^{(0), l}(0)=0$ for $j=1, \ldots, n$ (for $j=1$ the equality holds by assumption). Now Lemma 8 ends the proof.

Proof of Proposition 3. This is an immediate consequence of Lemma 9. 
Proof of Theorem 1. Let $\Gamma^{(0), 1}$ be the second order connection on $\mathbb{R}^{m, n}$ as in the proof of Lemma 7. Let $\mathcal{E}^{a}: J^{2} \rightsquigarrow S^{2} T^{*} \otimes V V^{A}$ be the operators from Example 2 for $a \in A$. Let $\left(a_{\nu}\right)$ be a basis of $A$, and let $\left(v^{l}\right)$ correspond to $\left(a_{\nu}\right)$ (as at the beginning of Section 3 ). One can show by a standard argument that the numbers $\Phi_{0}^{l}\left(\Gamma^{(0), 1}\right)=a_{1}^{(0), l}(0)($ see $(*))$ for $\mathcal{E}=\mathcal{E}^{a_{\nu}}$ are proportional (with a non-zero coefficient) to the Kronecker delta $\delta_{\nu}^{l}$. Hence the operators $\mathcal{E}^{a_{\nu}}$ are linearly independent. Theorem 1 is an immediate consequence of Propositions $1-3$ by a dimension argument.

\section{References}

[1] A. Cabras and I. Kolár, Prolongations of second order connections to vertical Weil bundles, Arch. Math. (Brno) 37 (2001), 333-347.

[2] M. Doupovec and I. Kolář, Iteration of fiber product preserving bundle functors, Monatsh. Math. 134 (2001), 39-50.

[3] M. Doupovec and W. M. Mikulski, Holonomic extension of connections and symmetrization of jets, Rep. Math. Phys., to appear.

[4] I. Kolár̆, The contact of spaces with connections, J. Differential Geom. 7 (1972), 563-570.

[5] I. Kolár̆, P. Michor and J. Slovák, Natural Operations in Differential Geometry, Springer, 1993.

[6] I. Kolář and W. M. Mikulski, Natural lifting of connections to vertical bundles, Rend. Circ. Mat. Palermo (2) Suppl. 63 (2000), 97-102.

Institute of Mathematics

Maria Curie-Skłodowska University

Pl. Marii Curie-Skłodowskiej 1

20-031 Lublin, Poland

E-mail: kurek@golem.umcs.lublin.pl
Institute of Mathematics Jagiellonian University Reymonta 4 30-059 Kraków, Poland E-mail: mikulski@im.uj.edu.pl

Received 4.3.2007

and in final form 6.6.2007 